\title{
Detecting Patterns in 5' Untranslated Regions of Genes Involved in the Regulation of Blood Pressure and Oxidative Stress
}

\author{
${ }^{1}$ Maria Sanchez-Aguilar, ${ }^{1}$ Luz Ibarra-Lara, ${ }^{2}$ Beatriz Zamora-Lopez, \\ ${ }^{1}$ Alicia Sanchez-Mendoza and ${ }^{3}$ Absalom Zamorano-Carrillo \\ ${ }^{1}$ Department of Pharmacology, National Institute of Cardiology “Ignacio Chávez”, Mexico City, Mexico \\ ${ }^{2}$ Department of Neurosciences, Faculty of Medicine, National Institute of Perinatology, \\ "Isidro Espinosa de los Reyes" and Department of Mental Health, UNAM, Mexico City, Mexico \\ ${ }^{3}$ Department of Molecular Biomedicine and Doctoral Program of Biotechnology, \\ Laboratory of Computational Biophysics and Biochemistry, ENMH-IPN, Mexico City, Mexico
}

Received 2013-08-26; Revised 2013-09-18; Accepted 2013-11-27

\begin{abstract}
Hypertension is a chronic physical condition with multifactorial causes. Although genetic factors have been associated to this pathological condition, characterization of the sequence patterns in the regulatory sites, for example, in 3' or 5' Untranslated Regions (UTR), remains to be explored. In particular, the 5' UTR of genes associated with the regulation of blood pressure and oxidative stress are analyzed in this article. To gain insight into how certain DNA motifs are involved with high blood pressure, we decided to perform a study with sequences from genes reported as regulators of blood pressure and oxidative stress. 5' UTR gene sequences were submitted to patterns recognition by the Multiple Em for Motif Elicitation (MEME) software. Afterward, the motifs obtained were searched for in the Transcription Element Search System (TESS) and Consite platforms, in order to identify the submitted sequence as element response of some transcription factor described previously. Three different motifs in each group of vasorelaxing-and vasocontractile-related gene sequences were detected. In the vasorelaxing group, motif lengths were 39 to 50 nucleotides and were located from -361 to -167 bp before the Open Reading Frame (ORF). In turn, motifs in vasocontractile group sequences were located from -619 to -570 nucleotides, with a length of from 18 to 40 nucleotides. Regarding the nucleic acid content in the motifs found, adenine was more prevalent in vasorelaxing-related sequences with $45 \%$ of the average frequency, whereas guanine on those vasocontractile-related sequences with $38 \%$. Distinct motif sequences and variations of nucleotide content in the promoter region of vasorelaxing and vasocontractile-activity related genes were detected. These motifs of each group of genes, with a putative antagonistic role between them, might be a differential cisregulatory elements of transcriptional machinery.
\end{abstract}

Keywords: Blood Pressure, Genes, Motifs, Transcription

\section{INTRODUCTION}

Blood Pressure (BP) is a physiological parameter defined as the force exerted by blood against any unit area of the vessel wall. Elements such as Heart Rate (HR) and Vascular Resistance (VR) are key points to control this parameter (Chen et al., 2007; Mukkamala et al., 2006).

Biological negative feedback systems regulate blood pressure by the activity of cardiac frequency, vascular resistance and blood volume. These physiological modulators are regulated by some hormones. Laboratory of Computational Biophysics and Biochemistry, ENMH-IPN, Mexico City, Mexico 
Specifically, metabolites such as adrenaline, noradrenaline, vasopressin, angiotensin II, dopamine and Reactive Oxygen Species (ROS) promote the elevation of blood pressure. On the contrary, the metabolic activity of enzymes such as catalase, glutathione peroxidase, hemooxygenase and endothelial nitric oxide synthase reduce it. Also, the expression of some receptors (e.g., bradykinin $\mathrm{B}_{2}$, adenosine $\mathrm{A}_{2}$, peroxisome proliferator activated receptor gamma) and hormones (brain natriuretic peptide and acetylcholine) is associated to lower blood pressure. All these molecules maintain the blood pressure in homeostatic conditions (Feletou et al., 2010; Vanhoutte, 2006).

Arterial Hypertension (HTA) is defined as the presence of systolic BP values $\geq 140 \mathrm{mmHg}$ and/or diastolic BP values $\geq 90 \mathrm{mmHg}$. Along with other vascular diseases, HTA is a risk factor for cardiovascular disease (Peralta et al., 2005; Velazquez-Monroy et al., 2002).

Hypertension elicits two different kinds of diffuse structural changes in the systemic microcirculation. Although several causes have been related with increased BP, various research groups have focused their investigations on determining whether genetic mechanisms serve as initial triggers in the genesis of the disease (Renna et al., 2013; Zenteno and Kofman, 2003). Considering the genetic susceptibility of HTA, it has been reported that the classical mendelian rules are not fully applied in the case of BP control, whereby the hypertension shows a complex model inheritance which requires different experimental and theoretical approaches for a better study. In this vein, it has been reported that the heritability of blood pressure or the genetic contribution is able to modulate the arterial tension in a $30-50 \%$ of its value (Doris, 2011; Timberlake et al., 2001).

Currently, different molecular techniques allow identify genes associated with pathological conditions suggesting their physiological role. Experimental studies such as linkage studies, candidate genes analysis, punctual mutations, Single Nucleotide Polymorphisms (SNPs) detection and recently bioinformatical analysis provide information for these characterizations (Kunes and Zicha, 2009; Zenteno and Kofman, 2003).

Motif discovery in sequences of interest, as regulatory regions of the gene expression, has been a strategy to find patterns with high information content that play a physiological role. An analysis of Perera et al. (2006) proposed novel PPAR gamma target genes in adipocytes by microarray profiling and computational detection of motifs in the 5' UTR of 182 related genes. Furthermore, they found 26 highly conserved sequences and identified a drosophila serpent homolog gene that has been reported as a participant in the fat body formation (Perera et al., 2006). Similarly an Motif Discovery Scan (MDscan), Siersbaek et al. (2010) found that CCAAT/enhancer binding gen (c/EBP) has a binding motif target of PPAR gamma transcription factor, additional to DR-1 binding element. Suggesting that these adipogenic factors function together as common site to regulate genes (Siersbaek et al., 2010). In this work, we performed an in silico analysis of the 5' UTR of genes encoding enzymes or proteins related with blood pressure regulation pathways. In other words, we identified patterns of sequences into the promoter regions of transcription factors that could be investigated as modulators of blood pressure.

\section{MATERIALS AND METHODS}

The Ensembl databases (http://www.ensembl.org/Homo_sapiens/Gene/) were the repository of the 5'UTR sequences. FASTA format files with sequences spanning 200 or $600 \mathrm{bp}$ were obtained for the vasorelaxing and vasocontractile activity genes. For the first group, we considered catalase (cat), glutathione peroxidase $(g p x 1)$, superoxide dismutase $(\operatorname{sod} 1)$, hemooxigenase types 1 and 2 (hmox $1, h m o x 2)$, endothelial nitric oxide synthase (nos3), brain natriuretic peptide (nppb), beta adrenergic receptor type 2 (adbr2), bradykinin receptor type 2 (bdkrb2), adenosine receptor 2A (adora2a), muscarinic receptor type 3 (chrm3), dopamine receptor type $1(d r d 1)$, the PPAR gamma isoform (pparg), adenine triphosphate-sensitive potassium channel (kcnj8) and small/intermediate conductance calcium-activated potassium channels (kcnn3, kcnj1). Regarding vasocontractile genes, we screened the motifs in xanthin dehydrogenase $(x d h)$, lipoxigenase types 5 (alox5), 12 (alox 12) and 15 (alox15), voltage-gated potassium channel (cacng5), nicotinamide adenine dinucleotide phosphate dehydrogenase (NAD[P]H) oxidase subunits Nox1 (nox1), Nox4 (nox4), Gp91 (cybb), p47 (ingl) and p22 (dync1h1) (all of these of $600 \mathrm{bp}$ in lenght); also muscarinic receptor type 1 (chrm 1$)$, serotonin receptor type 2 ( $h$ tr $2 a$ ), alpha adrenergic receptor 2A (adra2a), dopamine receptor type $5(d r d 5)$, endothelin receptor type 1 (ednl), vasopressin (avprla) and cyclooxygenase type 2 (ptgs2) (all of these $2000 \mathrm{bp}$ in lenght). 
With these sequences, we performed in Multiple Em for Motif Elicitation (MEME) program (http://meme.sdsc.edu/meme/intro.html) a search of patterns named motifs. This program analyzes and detects sequences with characteristics such as DNA binding or protein interaction domains. Default conditions offered by the web server were utilized; these parameters are minimum and maximum number of nucleotides in the motif. After obtaining the motifs, these were loaded on TESS database (www.ebil.upenn.edu/cgi-bin/tess/tess) to investigate if the motifs have been reported as transcription factors, DNA binding sites, or if they interact with protein functional domains. Similarly, this search was performed into Consite database (http://asp.ii.uib.no:8090/cgi-bin/CONSITE/consite/) with same purpose. Also, a quantification of nucleotide content in the motifs was carried out. GraphPad Prism program software was used to plot the results.

\section{RESULTS}

Bioinformatics analysis showed three highly relevant motifs with a high percentage of homology in both groups of genes. For vasorelaxing-activity-relatedsequences, Motifs 1 and 2 were 50 nt in length and Motif 3 has a length of $39 \mathrm{nt}$. Positions of these motifs in reference to zero in the beginning of ORF was $-167,-199$ and -361 respectively (Table $\mathbf{1}$ ).

In the group of vasocontractile activity related sequences, the length for Motif 1 was $40 \mathrm{nt}$, for Motif 2 $20 \mathrm{nt}$ and for Motif 3, 18nt. Their positions were found at $-570,-619$ and $-582 \mathrm{nt}$ before the transcription site (Table 1). Afterward, we estimated the presence of each nucleotide (adenine, thymine, guanine and cytosine) in each motif found. In genes related with a vasorelaxing effect, adenine was present in $45 \%$, guanine in $30 \%$, thymine in $16 \%$ and cytosine in $9 \%$ (Fig. 1A). In contrast, in sequences of genes related to a vasocontractile effect, guanine appeared to be the most frequent nucleotide (38\%) followed by cytosine (29\%), thymine (31\%) and finally, adenine (1\%) (Fig. 1B).

According to these results the adenine content is the most evident factor that distinguishes the two studied groups.

To know whether motifs show a similarity with sequences reported as response elements of some characterized genes, the motifs were searched in TESS and Consite databases. With this analysis, a prediction about the affinity of some transcription factors for the motifs can be obtained and as consequence proteins with a regulatory role in blood pressure can be proposed.

Among the motifs in vasorelaxing genes sequences, Motif 1 was found in several transcription factors (DBF4, ETF, TBP, TFIIA, TMF and YY1) corresponding to TESS database and the search in Consite database four more were included (SOX5, HFH2, HMG-IY and Evi-1). Motif 2 was associated with the TCF family proteins and LEF-1 according TESS database, whereas using Consite SOX5, HFH-2, HMGIY, HNF-1 and AML-1 were found. In particular, SOX5, HFH-2 and HMG-IY contain Motif 1 and 2 (Table 2) blue letters. Analysis on Motif 3 revealed its existence in MAZ, AP-2 $\alpha$, SP and H4TF-1 using TESS, Consite was unable to advise similarity with response element contained into that database. These results could be suggested as new transcription factor participating in the vascular relaxation (Table 2).
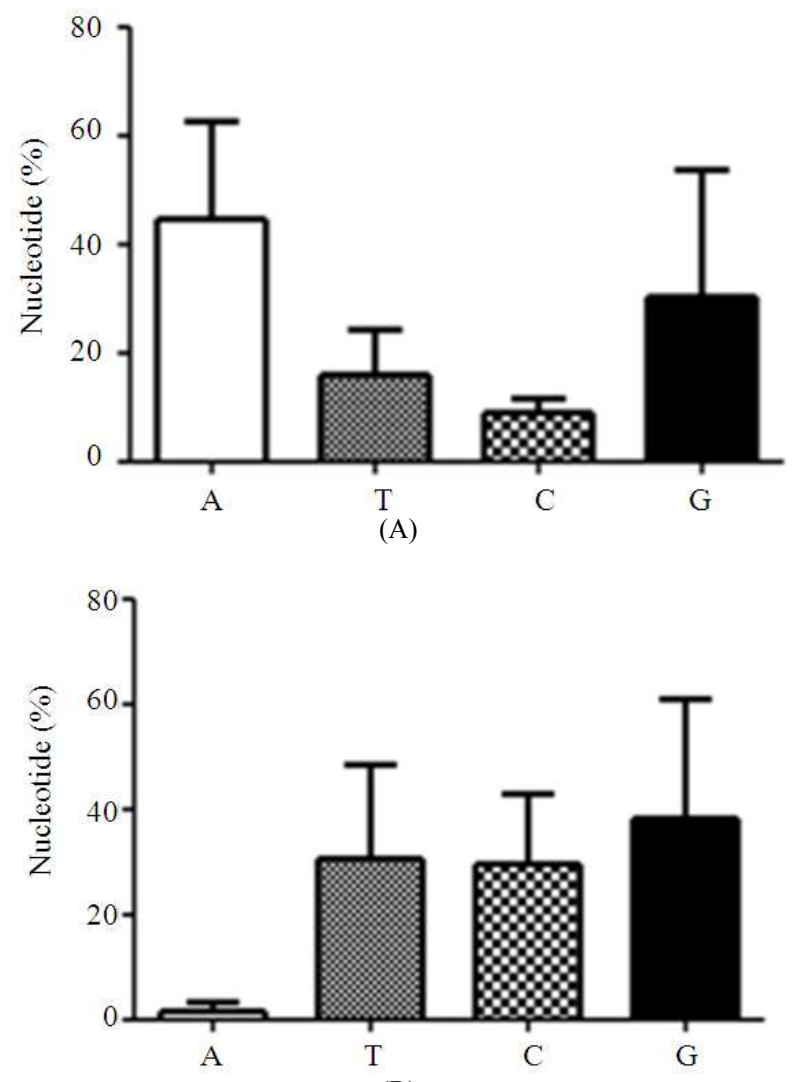

(B)

Fig. 1. Nucleotide content of the motifs found. (A) With a putative vasorelaxing activity; (B) with a putative vasocontractile activity. Bars represent mean +/- E.S 
Table 1. Motifs detected by MEME program into 5' UTR region of genes studied

\begin{tabular}{llc}
\hline $\begin{array}{l}\text { No. } \\
\text { of motif }\end{array}$ & Motifs with a putative vasorelaxing activity & Initial position \\
\hline 1 & AAAAAAAATTATACAAGTAAAATAATAATTATAAGAAAAAACAGTAAAAA & -167 \\
2 & AGAAAAAAAAAAGTACCGAAATATGCTTTCATTAAAAATTATTCTAAAAT & -199 \\
3 & GGGGAGGGGGGGGGAGGGACGGGGGGGGAGGGCCGGGCC & -361 \\
& Motifs with a putative vasocontractile activity & -570 \\
1 & TGCTGGGGGGGCGGGGGGGGGGGCGGGGGCGGGGGCGGGG & -619 \\
2 & TTTGTTTCTTTTCTCAGC & -582 \\
3 & CCTGCCTCCCTCTCCGGG & \\
\hline
\end{tabular}

Table 2. Transcription factors found in the TESS and Consite databases containing the motifs found by MEME

\begin{tabular}{|c|c|c|c|c|c|}
\hline \multicolumn{6}{|c|}{ Vasorelaxing Activity related Genes } \\
\hline \multicolumn{2}{|l|}{ Motif 1} & \multicolumn{2}{|l|}{ Motif 2} & \multicolumn{2}{|l|}{ Motif 3} \\
\hline TESS & Consite & TESS & Consite & TESS & Consite \\
\hline DBF4 & SOX5 & TCF-2 $2 \alpha$ & SOX5 & MAZ & Any \\
\hline ETF & HFH-2 & TCF $-1^{\mathrm{a}}$ & HFH-2 & $\mathrm{AP} 2 \alpha$ & \\
\hline TBP & HMG-IY & TCF-1B & HMG-IY & SP & \\
\hline TFIIA & Evi-1 & LEF-1 & HNF-1 & H4TF-1 & \\
\hline TMF & & & AML-1 & & \\
\hline \multicolumn{6}{|l|}{ YY1 } \\
\hline Motif 1 & Motif 2 & Motif 3 & & & \\
\hline SP-1 & RREB-1 & $\mathrm{TCF}-2 \alpha$ & FREAC-2 & Any & Any \\
\hline AP-1 & & LEF-1 & Irf-1 & & \\
\hline ETF & & & SOX-5 & & \\
\hline$E R \alpha$ & & & HMG-IY & & \\
\hline
\end{tabular}

Regarding motifs found into genes related to vasocontractile responses, we discovered a similar pattern. TESS analysis showed that Motif 1 is present in $\mathrm{SP} 1, \mathrm{AP}-1, \mathrm{ER} \alpha$ and ETF, this last response element was also found in vasorelaxing Motif 1 (Table 2), on the top, green letters. Consite database search showed a correspondence with RREB-1 for this motif. TESS analysis, for the Motif 2, provided TCF-2 $\alpha$ and LEF-1, while Consite paired it with SOX5, HMG-IY, FREAC2 and Irf-1. In the case of Motif 3, similarity was undetectable for both databases. Particularly, SOX5 and HMG-IY were another response elements that contain the Motif 3 obtained from vasorelaxing activity genes (Table 2) below, green letters. Therefore, we could suggest that these motifs are putative binding sites of transcription factors that might have a regulatory role in blood pressure.

\section{DISCUSSION}

In silico studies on biomolecules permit to detect information that could be relevant for their biochemical functions. Although, several threedimensional models of DNA have been reported, mainly DNA analysis has been almost limited to understand the combinations of nucleotides in sequences that contain instructions about the cellular machinery. The interest in the information content in key regions has driven the design of algorithms to excavate the mechanisms of data transmission between biomolecules. In particular, flanking regions of ORF are of interest due to their regulatory role. The study of characteristic patterns of group of sequences is a route to this end. In this work, we performed a search of patterns in 5'UTR sequences of vasorelaxing and vasocontractile genes, through the MEME program and a subsequent search into TESS and Consite databases.

The results of the present analysis show that MEME platform allows the identification putative response in genomic sequences that could be related to blood pressure. By discovering of sequence motif, a short pattern of nucleotides can be deemed to have biological significance (Spontaneo and Cercone, 2011). In addition, this platform is a tool that enables a better characterization of target genes (Bailey et al., 2009). As an example, the study developed by Perera et al. (2006) permitted to predict novel target genes for PPAR gamma in human cultured adipocytes. They identified three 
important genes for fat body composition in drosophila. Because some of them were not found in the transcription database TFBIND, the authors concluded that these motifs could represent novel transcription factors (Perera et al., 2006). The length of DNA motifs sequences suggests that the information content in this number of nucleotides facilitates the spatial availability of DNA. However, some transcription factors exhibit flexibility to bind a few binding sites or bind DNA in some configuration (Reid et al., 2010). In this work, we found that in vasorelaxing group, the motif length was higher (50 nt) with respect to those detected in the vasocontractile group (18 to $40 \mathrm{nt}$ ). Besides, due to vasorelaxing sequences are closer to the transcription starting point ( $-240 \mathrm{bp}$ on average) than vasocontractile sequences (-590 bp on average), both could have different molecular crowding for the transcription, or distinct interaction modes with cisregulation elements (Gruel et al., 2011).

In our results, nucleotide content of regions was a parameter that distinguishes the vasorelaxing motifs from vasocontractile. Adenine showed the highest proportion $(45 \%)$ in the first group, whereas guanine was more abundant $(38 \%)$ in the second, possibly evidencing a regulatory function. Interestingly, it has been suggested that the high content of specific nucleotides could be a point for control of the function. Specifically, guanine-rich sequence in 5'-UTRs of RNA oncogenic can regulate negatively the activity of the telomerase through G-quadruplex formation at the telomeric end (Kaushik et al., 2011; Patel et al., 2007). Therefore, the presence of adenine-rich or guanine-rich regions in the motifs found might be a molecular signal for the differential transcription of genes in distinct contexts related to blood pressure.

\section{CONCLUSION}

The information in 5, UTR sequences could be a key factor in the gene expression of proteins that regulate the arterial pressure. Although more studies are required, the motifs reported in this work and the content adenine and guanine give rise to propose experimental and theoretical works that could take into account these findings.

\section{ACKNOWLEDGEMENT}

This research was funded by SIP-IPN (Project 20130466) and COFAA-IPN and SNI-CONACYT.

\section{REFERENCES}

Bailey, T.L., M. Boden, F.A. Buske, M. Frith and C.E. Grant et al., 2009. MEME suite: Tools for motif discovery and searching. Nucl. Acids Res., 37: W202-W208. DOI: 10.1093/nar/gkp335

Chen, X., J.K. Kim, J.A. Sala-Mercado, R.L. Hammond, G. Swamy et al., 2007. Identification of the total peripheral resistance baroreflex impulse response from spontaneous hemodynamic variability: Validation by chronic arterial baroreceptor denervation. Proceedings of the 29th Annual International Conference of the IEEE Engineering in Medicine and Biology Society, Aug. 22-26, IEEE Xplore Press, Lyon, pp: 1051-1054. DOI: 10.1109/IEMBS.2007.4352475

Doris, P.A., 2011. The genetics of blood pressure and hypertension: The role of rare variation. Cardiovascular Therapeut., 29: 37-45. DOI: 10.1111/j.1755-5922.2010.00246.x

Feletou, M., R. Kohler and P.M. Vanhoutte, 2010. Endothelium-derived vasoactive factors and hypertension: Posible roles in pathogenesis and as treatment targets. Curr. Hypertens Res., 12: $267-$ 275. PMID: 20532699

Gruel, J., M. LeBorgne, N. LeMeur and N. Theret, 2011. Simple Shared Motifs (SSM) in conserved region of promoters: A new approach to identify co-regulation patterns. BMC Bioinform., 12: 365-378. DOI: 10.1186/1471-2105-12-365

Kaushik, M., S. Kauchik, A. Bansal, S. Saxena, S. Kukreti, 2011. Structural diversity and specific recognition of four stranded G-quadruplex DNA. Curr. Mol. Med., 11: 744-769. PMID: 21999150

Kunes, J. and J. Zicha, 2009. The interaction of genetic and environmental factors in the etiology of hypertension. Physiol. Res., 58: S33-S41. PMID: 20131935

Mukkamala, R., J.K. Kim, Y. Li, J. Sala-Mercado, R.L. Hammond et al., 2006. Estimation of arterial and cardiopulmonary total peripheral resistance baroreflex gain values: Validation by chronic arterial baroreceptor denervation. Am. J. Physiol. Heart Circ. Physiol., 290: H1830-H1836. PMID: 16306216

Patel, D.J., A.T. Phan and V. Kuryavyi, 2007. Human telomere, oncogenic promoter and 5'-UTR Gquadruplexes: Diverse higher order DNA and RNA targets for cancer therapeutics. Nucleic Acids Res., 35: 7429-7455. DOI: 10.1093/nar/gkm711 
Peralta, M.R., A.L. Esqueda, G.P. Hernandez, O.V. Monroy and J.M. Reding et al., 2005. National Resurvey of Arterial Hypertension (RENAHTA). mexican consolidation of the cardiovascular risk factors. national follow-up cohort. Arch. Cardiol. Mex., 75: 96-111. PMID: 15909748

Perera, R.J., E.G. Marcusson, S. Koo, X. Kang and Y. Kim et al., 2006. Identification of novel PPAR $\gamma$ target genes in primary human adipocytes. Gene, 369: 90-99. DOI: 10.1016/j.gene.2005.10.021

Reid, J.E., KJ. Evans, N. Dyer, L. Wernisch and S. Ott, 2010. Variable structure motifs for transcription factor binding sites. BMC Genom., 11: 30-48. DOI: 10.1186/1471-2164-11-30

Renna, N.F., N. de Las Heras and R.M. Miatello, 2013. Pathophysiology of vascular remodeling in hypertension. Int. J. Hypertens. DOI: $10.1155 / 2013 / 808353$

Siersbaek, R., R. Nielsen and S. Mandrup, 2010. PPAR $\gamma$ in adipocyte differentiation and metabolism-novel insights from genome-wide studies. FEBS Lett., 584: 3242-3249. PMID: 20542036
Spontaneo, L. and N. Cercone, 2011. Correlating CpG islands, motifs and sequence variants in human chromosome 21. BMC Genom., 12: S10-S18. DOI: 10.1186/1471-2164-12-S2-S10

Timberlake, D.S., D.T. O’Connor and R.J. Parmer, 2001. Molecular genetics of essential hypertension: Recent results and emerging strategies. Curr. Opin. Nephrol. Hypertens., 10: 71-79. PMID: 11195056

Vanhoutte, P.M., 2006. Endothelial dysfunction and vascular pathology. Bull. Mem. Acad. R Med. Belg, 161: 529-536. PMID: 17503729

Velazquez-Monroy, O., M. Rosas-Peralta, A. LaraEsqueda, G. Pastelin-Hernandez and F. Attie et al., 2002. Hipertensión arterial en Mexico: Resultados de la Encuesta Nacional de Salud (ENSA) 2000. Arch. Cardiol. Mexico, 72: 71-84. PMID: 11933703

Zenteno, J.C. and S. Kofman-Alfaro, 2003. Aspectos geneticos de la hipertensión arterial. Rev. Med. Hospital General Mexico, 66: 218-223. 